\title{
New short regimens for latent tuberculosis treatment: safety first!
}

\author{
Dick Menzies ${ }^{1}$ and Anete Trajman ${ }^{2,3}$
}

\begin{abstract}
Affiliations: ${ }^{1}$ Respiratory Epidemiology and Clinical Research Unit, Montreal Chest Institute, Montreal, QC, Canada. ${ }^{2}$ Federal University of Rio de Janeiro, Rio de Janeiro, Brazil. ${ }^{3}$ McGill University, Montreal, QC, Canada.

Correspondence: Anete Trajman, Federal University of Rio de Janeiro, Rua Macedo Sobrinho 74/203, Humaitá, Rio de Janeiro 22271-080, Brazil. E-mail: atrajmanđggmail.com
\end{abstract}

@ERSpublications

Safety must be the first consideration in evaluating any new regimen for latent tuberculosis treatment. Caution is needed when using isoniazid plus rifapentine, once or twice weekly, in individuals aged over 50 years, even for a short period. http://ow.ly/T0fT30mOs7m

Cite this article as: Menzies D, Trajman A. New short regimens for latent tuberculosis treatment: safety first! Eur Respir J 2018; 52: 1802180 [https://doi.org/10.1183/13993003.02180-2018].

Prevention of active tuberculosis (TB) through treatment of latent tuberculosis infection (LTBI) has substantial potential individual and public health benefits, and hence is a cornerstone to TB elimination $[1,2]$. Yet, only a small proportion of those who might benefit from LTBI treatment will start it, and an even smaller proportion will complete it [3]. In 1970, the American Thoracic Society recommended isoniazid preventive therapy (IPT) based on strong evidence, from multiple randomised trials, of its safety and efficacy for TB prevention [4]. The more widespread use of IPT was followed, within a year, by reports of fatal hepatotoxicity [5]. This has continued to be the Achilles' heel of IPT: although fatality rates are now low, "liver deaths" continue to occur with IPT [6]. 60 years later, awareness about this drawback is widespread among providers and patients, resulting in low rates of prescription [3] and acceptance [7]. The experience with a 2-month regimen of rifampin with pyrazinamide in 2000-2001 [8], with more fatal and near-fatal cases of drug-induced liver disease, was another setback for TB prevention efforts. Safety is an essential pre-requisite for any TB preventive regimen, since LTBI is an asymptomatic, non-contagious condition that progresses to disease in only a minority of affected individuals. Judged against the safety record of anti-hypertensives or lipid-lowering agents, isoniazid appears unacceptably and alarmingly toxic.

More recently, mostly over the past decade, several large scale trials have demonstrated that three rifamycin-containing regimens (three months once weekly isoniazid plus rifapentine (3HP) [9-11], 3-4 months daily isoniazid plus rifampin (3-4HR) [12], and 4 months daily rifampin (4R) $[11,13,14])$ achieve better completion rates and similar preventive efficacy compared to isoniazid alone [15]. However, of these three regimens, only $4 \mathrm{R}$ has been consistently safer than isoniazid in trials $[11,13-15]$ and in observational studies [16, 17], while $3 \mathrm{HR}$ has similar safety as $6 \mathrm{H}$ [12] and $3 \mathrm{HP}$ results in less hepatotoxicity, but other serious adverse events have been reported $[9,10]$. While children tolerate all these regimens very well $[13,18]$, older adults are at the highest risk for adverse events. It is thus crucial to evaluate the safety of new LTBI treatment regimens in older adults.

In this issue of the European Respiratory Journal, GAO et al. [19] report very high rates of adverse events in interferon- $\gamma$ release assay (IGRA)-positive older adults (aged 50-70 years) who were randomised to receive one of two short rifapentine-containing regimens: 3HP or 2 months of twice weekly rifapentine $600 \mathrm{mg}$ plus isoniazid $600 \mathrm{mg}$. The original protocol had to be interrupted prematurely due to the high rate of adverse events, although the details are not fully reported. This occurred despite the pre-randomisation 
exclusion of individuals with hepatic disease, and despite participants only receiving, at most, 8 and 6 weeks of treatment, respectively, instead of the originally proposed 12 and 8 weeks. Thus, modified (shorter) rifapentine-containing regimens were actually evaluated. The authors claim "fairly high efficacy" of both tested regimens, although their data do not confirm a significant reduction of risk of active TB with either regimen. Moreover, potential for bias in this study is high [20]. First, misclassification bias might have occurred, as almost all active $\mathrm{TB}$ cases were clinically diagnosed, without bacteriological confirmation. Secondly, a positive IGRA was both an inclusion criterion and part of the criteria for active TB. Additionally, some of the investigators who made end-point determinations were unblinded, resulting in high risk of detection bias. Finally, attrition bias might have occurred, since patients who abandoned treatment or were lost to follow-up were excluded from the analyses. However, since data from other well conducted trials have shown excellent efficacy of $3 \mathrm{HP}[9,10,18]$, the question of whether the efficacy findings in this trial are real is less relevant.

The main lesson from this study is the caution needed when using isoniazid plus rifapentine, once or twice weekly, in individuals aged over 50 years, even for a short period. While the methodological limitations of this trial reduce its reliability, these toxicity findings do raise concerns about the use of rifapentine plus isoniazid regimens in older adults, and warrant further careful evaluation.

Safer regimens to treat LTBI are definitely needed. Safety must be the first consideration in evaluating any new, short rifamycin-based regimen if we are to achieve the level of LTBI therapy uptake necessary to have a substantial impact on the current TB epidemic.

Conflict of interest: None declared.

\section{References}

1 World Health Organization. Latent tuberculosis infection: updated and consolidated guidelines for programmatic management. Geneva, World Health Organization, 2018. Available from: http://www.ncbi.nlm.nih.gov/books/ NBK531235/ Date last accessed: November 23, 2018.

2 Dye C, Glaziou P, Floyd K, et al. Prospects for tuberculosis elimination. Annu Rev Public Health 2013; 34: 271-286.

3 Alsdurf H, Hill PC, Matteelli A, et al. The cascade of care in diagnosis and treatment of latent tuberculosis infection: a systematic review and meta-analysis. Lancet Infect Dis 2016; 16: 1269-1278.

4 Smieja MJ, Marchetti CA, Cook DJ, et al. Isoniazid for preventing tuberculosis in non-HIV infected persons. Cochrane Database Syst Rev 2000; 2: CD001363.

5 Garibaldi RA, Drusin RE, Ferebee SH, et al. Isoniazid-associated hepatitis. Report of an outbreak. Am Rev Respir Dis 1972; 106: 357-365.

6 Kabbara WK, Sarkis AT, Saroufim PG. Acute and fatal isoniazid-induced hepatotoxicity: a case report and review of the literature. Case Rep Infect Dis 2016; 2016: 3617408

7 Sumartojo E. When tuberculosis treatment fails. A social behavioral account of patient adherence. Am Rev Respir Dis 1993; 147: 1311-1320.

8 Centers for Disease Control and Prevention (CDC), American Thoracic Society. Update: adverse event data and revised American Thoracic Society/CDC recommendations against the use of rifampin and pyrazinamide for treatment of latent tuberculosis infection - United States, 2003. MMWR Morb Mortal Wkly Rep 2003; 52: 735-739.

9 Sterling TR, Villarino ME, Borisov AS, et al. Three months of rifapentine and isoniazid for latent tuberculosis infection. N Engl J Med 2011; 365: 2155-2166.

10 Martinson NA, Barnes GL, Moulton LH, et al. New regimens to prevent tuberculosis in adults with HIV infection. N Engl J Med 2011; 365: 11-20.

11 Menzies D, Adjobimey M, Ruslami R, et al. Four months of rifampin or nine months of isoniazid for latent tuberculosis in adults. N Engl J Med 2018; 379: 440-453.

12 Geijo MP, Herranz CR, Vaño D, et al. Short-course isoniazid and rifampin compared with isoniazid for latent tuberculosis infection: a randomized clinical trial. Enferm Infecc Microbiol Clin 2007; 25: 300-304.

13 Diallo T, Adjobimey M, Ruslami R, et al. Safety and side effects of rifampin versus isoniazid in children. $N$ Engl J Med 2018; 379: 454-463.

14 Menzies D, Long R, Trajman A, et al. Adverse events with 4 months of rifampin therapy or 9 months of isoniazid therapy for latent tuberculosis infection: a randomized trial. Ann Intern Med 2008; 149: 689-697.

15 Stagg HR, Zenner D, Harris RJ, et al. Treatment of latent tuberculosis infection: a network meta-analysis. Ann Intern Med 2014; 161: 419-428.

16 Lardizabal A, Passannante M, Kojakali F, et al. Enhancement of treatment completion for latent tuberculosis infection with 4 months of rifampin. Chest 2006; 130: 1712-1717.

17 Page KR, Sifakis F, Montes de Oca R, et al. Improved adherence and less toxicity with rifampin vs isoniazid for treatment of latent tuberculosis: a retrospective study. Arch Intern Med 2006; 166: 1863-1870.

18 Villarino ME, Scott NA, Weis SE, et al. Treatment for preventing tuberculosis in children and adolescents: a randomized clinical trial of a 3-month, 12-dose regimen of a combination of rifapentine and isoniazid. JAMA Pediatr 2015; 169: 247-255.

19 Gao L, Zhang H, Xin H, et al. Short-course regimens of rifapentine plus isoniazid to treat latent tuberculosis infection in older Chinese patients: a randomised controlled study. Eur Respir J 2018; 52: 1801470.

20 Higgins JPT, Altman DG, Gøtzsche PC, et al. The Cochrane Collaboration's tool for assessing risk of bias in randomised trials. BMJ 2011; 343: d5928. 